\title{
A new classification for hepatocellular carcinoma with hepatic vein tumor thrombus
}

\author{
Zhen-Hua Chen ${ }^{1,2 \#}$, Kang Wang ${ }^{1 \#}$, Xiu-Ping Zhang ${ }^{1,3 \#}$, Jing-Kai Feng ${ }^{1}$, Zong-Tao Chai ${ }^{1}$, Wei-Xing Guo ${ }^{1}$, \\ Jie Shi ${ }^{1}$, Meng-Chao $\mathrm{Wu}^{1,2}$, Wan Yee Lau ${ }^{2,4}$, Shu-Qun Cheng ${ }^{1,2}$ \\ ${ }^{1}$ Department of Hepatic Surgery VI, Eastern Hepatobiliary Surgery Hospital, Second Military Medical University, Shanghai, China; ${ }^{2}$ The National \\ Research Cooperative Group for Diagnosis and Treatment of Hepatocellular Carcinoma with Tumour Thrombus, Shanghai, China; ${ }^{3}$ Department of \\ Hepatobiliary and Pancreatic Surgical Oncology, The First Medical Center of Chinese People's Liberation Army (PLA) General Hospital, Beijing, \\ China; ${ }^{4}$ Faculty of Medicine, The Chinese University of Hong Kong, Shatin, Hong Kong, China \\ Contributions: (I) Conception and design: SQ Cheng, WY Lau, ZH Chen, K Wang, XP Zhang, MC Wu; (II) Administrative support: SQ Cheng; \\ (III) Provision of study materials or patients: ZT Chai, WX Guo, J Shi; (IV) Collection and assembly of data: ZH Chen, K Wang, XP Zhang; \\ (V) Data analysis and interpretation: ZH Chen, K Wang, JK Feng; (VI) Manuscript writing: All authors; (VII) Final approval of manuscript: All \\ authors. \\ "These authors contributed equally to this work. \\ Correspondence to: Shu-Qun Cheng, MD, PhD. Eastern Hepatobiliary Surgery Hospital, Second Military Medical University, 225 Changhai Road, \\ Shanghai 200433, China. Email: chengshuqun@aliyun.com.
}

Background: Hepatic vein tumor thrombus (HVTT) is a significant poor risk factor for survival outcomes in hepatocellular carcinoma (HCC) patients. Currently, the widely used international staging systems for HCC are not refined enough to evaluate prognosis for these patients. A new classification for macroscopic HVTT was established, aiming to better predict prognosis.

Methods: This study included 437 consecutive HCC patients with HVTT who underwent different treatments. Overall survival (OS) and time-dependent receiver operating characteristic (ROC) curve area analysis were used to determine the prognostic capacities of the new classification when compared with the different currently used staging systems.

Results: The new HVTT classification was defined as: type I, tumor thrombosis involving hepatic vein (HV), including microvascular invasion; type II, tumor thrombosis involving the retrohepatic segment of inferior vena cava; and type III, tumor thrombosis involving the supradiaphragmatic segment of inferior vena cava. The numbers (percentages) of patients with types I, II, and III HVTT in the new classification were 146 (33.4\%), 143 (32.7\%), and 148 (33.9\%), respectively. The 1-, 2-, and 3-year OS rates for types I to III HVTT were $79.5 \%, 58.6 \%$, and $29.1 \% ; 54.8 \%, 23.3 \%$, and $13.8 \%$; and $24.0 \%, 10.0 \%$, and $2.1 \%$, respectively. The time-dependent-ROC curve area analysis demonstrated that the predicting capacity of the new HVTT classification was significantly better than any other staging systems.

Conclusions: A new HVTT classification was established to predict prognosis of HCC patients with HVTT who underwent different treatments. This classification was superior to, and it may serve as a supplement to, the commonly used staging systems.

Keywords: Hepatocellular carcinoma (HCC); hepatic vein tumor thrombus (HVTT); staging system

Submitted Jul 10, 2019. Accepted for publication Aug 29, 2019.

doi: 10.21037/hbsn.2019.10.07

View this article at: http://dx.doi.org/10.21037/hbsn.2019.10.07 


\section{Introduction}

Hepatocellular carcinoma (HCC) is the fifth most common cancer and the third leading cause of cancer-related death worldwide $(1,2)$. This tumor has a great tendency to involve major vascular structures, such as the portal vein $(\mathrm{PV})$, hepatic vein $(\mathrm{HV})$, and even the inferior vena cava (IVC). Although HCC involvement of $\mathrm{HV}$ is less frequently observed than $\mathrm{PV}$, tumor thrombus invading $\mathrm{HV}$ to extend to IVC and right atrium (RA) has been reported $(3,4)$. The American Association for the Study of Liver Diseases/Barcelona Clinic for Liver Cancer staging system and treatment guidelines classify HCC with macroscopic vascular invasion to be at an advanced stage of disease, and sorafenib is the only recommended treatment (5). Generally, the prognosis of HCC patients with hepatic vein tumor thrombus (HVTT) or inferior vena cava tumor thrombus (IVCTT) is extremely poor.

With recent improvements in surgical techniques, medical care, and non-surgical treatment, treatment modalities for these patients vary greatly among different institutions. A Japanese team conducting a research with a large series of HVTT patients $(6,7)$ recommended hepatic resection combined with thrombectomy to be the treatment of choice in selected HVTT patients. For patients with HVTT not suitable for surgery because of concomitant liver dysfunction, non-surgical treatment such as transcatheter arterial chemoembolization (TACE), radiotherapy, and/or systemic chemotherapy have been used (8-13). A specific and concise classification is urgently needed to compare the effectiveness of the diversity of treatment modalities for the different subgroups of HCC patients with HVTT.

Several staging systems, such as the TNM classification (14), the Cancer of the Liver Italian Program (CLIP) score (15), the Japan Integrated Staging (JIS) score (15), the Chinese University Prognostic Index (CUPI) (16), and the Barcelona Clinic Liver Cancer (BCLC) (17) staging systems have been developed for HCC to predict prognosis, to guide treatment, and to compare results of different treatments. Most of these staging systems, including the TNM, CLIP, JIS, and BCLC consider vascular invasion to be an important prognostic factor. However, they are not refined enough to evaluate prognosis in HCC patients with macroscopic HVTT. Kokudo et al. (6) proposed a specific classification for HCC with HVTT, which categorized HVTT as tumor thrombosis in a peripheral hepatic vein (pHVTT, Vv1), a major hepatic vein (mHVTT, Vv2), or inferior vena cava
(IVCTT, Vv3). Although this classification is easy and convenient to use, it is suboptimal in predicting prognosis of patients in the pHVTT ( $\left.\mathrm{V}_{\mathrm{v}} 1\right)$ and mHVTT ( $\left.\mathrm{V}_{\mathrm{v} 2}\right)$ groups, thus limiting its clinical applicability.

This study was conducted to establish a new and practical classification to better stratify and refine macroscopic HVTT to improve on its prediction in prognosis.

We present the following article in accordance with the STROBE reporting checklist (available at http://dx.doi. org/10.21037/hbsn.2019.10.07).

\section{Methods}

\section{Diagnostic criteria for HVTT and PVTT}

The presence of HVTT and/or PVTT was diagnosed using radiologic imagings (CT/MRI/ultrasound) and/or histopathology (18). HVTT was classified into 3 grades: type I: tumor thrombosis involving $\mathrm{HV}$, including microvascular invasion; type I was further subdivided into type Ia: tumor thrombosis involving the peripheral $\mathrm{HV}$, and type $1 \mathrm{~b}$ : tumor thrombosis involving the major HV; type II: tumor thrombosis involving the retrohepatic segment of inferior vena cava; and type III: tumor thrombosis involving the supradiaphragmatic segment of inferior vena cava; type III was further subdivided into type IIIa: tumor thrombosis advancing into the RA and type IIIb: tumor thrombosis not advancing into the RA (Table 1 and Figure 1). PVTT was classified into the 4 grades according to the extent of PVTT in the PV based on Cheng's Classification (19): type I, tumor thrombus in the segmental branches of the PV or above; type II, tumor thrombus extending to the right or the left PV; type III, tumor thrombus extending to the main $\mathrm{PV}$; and type IV, tumor thrombus extending to the main PV and the superior mesenteric vein.

\section{Inclusion criteria}

The inclusion criteria were patients with: (I) age between 18 and 75 years; (II) Child-Pugh class A or selected B liver function (score $\leq 7$ ); (III) HCC with macroscopic HVTT or IVCTT; (IV) no extrahepatic or distant metastases; (V) absence of macroscopic bile duct tumor thrombus, and (VI) no other associated malignancies.

\section{Study population}

This study included 437 consecutive HCC patients with 
Table 1 Classification of HVTT

\begin{tabular}{ll}
\hline Types & Subtypes \\
\hline Type I: tumor thrombosis formation under microscopy & \\
Type I: tumor thrombosis involving the hepatic vein & Type la: tumor thrombosis involving the peripheral hepatic vein \\
& Type Ib: tumor thrombosis involving the major hepatic vein \\
$\begin{array}{l}\text { Type II: tumor thrombosis involving the retrohepatic segment of the } \\
\text { inferior vena cava }\end{array}$ & Type III: tumor thrombosis involving the supradiaphragmatic segment tumor thrombosis not advancing into the right atrium \\
of the inferior vena cava & Type IIlb: tumor thrombosis advancing into the right atrium
\end{tabular}

HVTT, hepatic vein tumor thrombus.
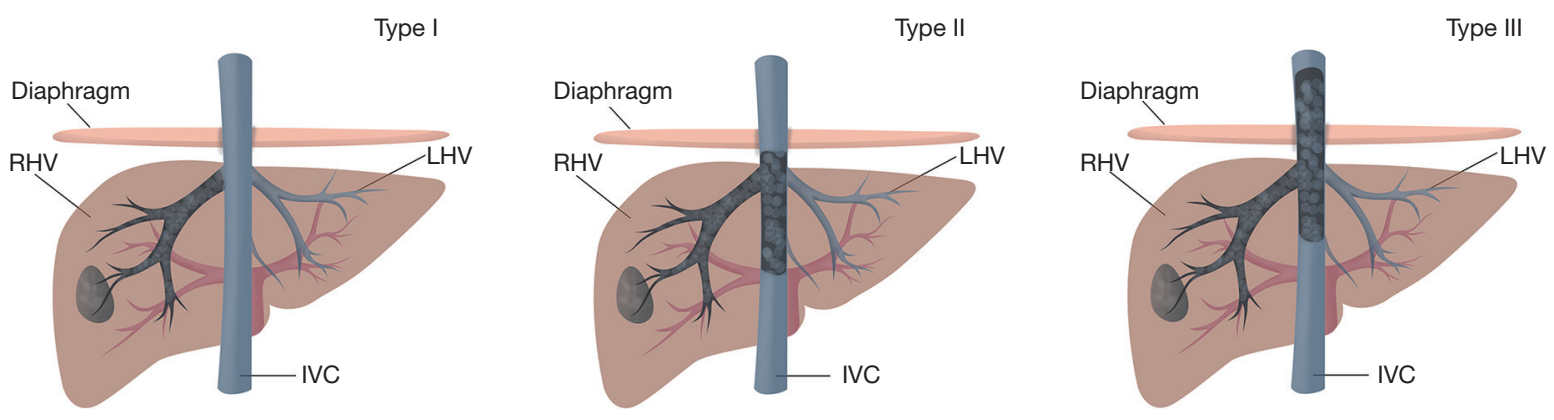

Figure 1 Classification of hepatic vein tumor thrombus. RHV, right hepatic vein; LHV, left hepatic vein; IVC, inferior vena cava.

HVTT or IVCTT who underwent treatments, including liver resection (LR), transcatheter chemoembolization (TACE), TACE combined with radiotherapy (TACE-RT), or best supportive care (BSC) from January 2008 to January 2016 at the Eastern Hepatobiliary Surgery Hospital. The demographic, clinical and pathological data and survival status of the patients were recorded prospectively and entered into an HCC database and analyzed retrospectively. The included patients were divided into 3 subgroups according to the New Classification for HVTT (types I-III). The present study was performed in accordance with the Declaration of Helsinki (as revised in 2013). The study was approved by the Eastern Hepatobiliary Surgery Hospital Research Ethics Committee (No. EHBHKY-2017-002-032). Informed consent was obtained from all the patients prior to treatment, and for their data to be used for research purposes.

\section{Treatment}

All patients were assessed by a multidisciplinary team of experienced liver surgeons, oncologists, radiologists and hepatologists at the Eastern Hepatobiliary Surgery Hospital. Surgical resection was the treatment of choice if the disease was resectable. The surgical procedures were reported previously $(13,20,21)$. Only patients with liver functions of Child-Pugh A or selected B were offered surgery. After exploration, routine intraoperative ultrasonography was carried out to determine the location and extent of HVTT, IVCTT, PVTT, and to rule out any preoperatively undetected tumors in the future liver remnant. The abdominal cavity was carefully searched for the extent of local disease, extrahepatic metastases, and peritoneal seeding. For HVTT, the tumor thrombus was either resected en bloc with the LR or it was extracted out of the vascular lumen depending on its location and extent. For HVTT with a tumor thrombus extending to the IVC, the infrahepatic and suprahepatic IVC were exposed and encircled with umbilical tapes for total hepatic vascular exclusion (THVE). Before the initiation of THVE, test clamping of the IVC was repeated several times to determine whether a venovenous bypass was necessary. During THVE, the patient's hemodynamic condition was carefully monitored and aggressively treated by the anesthesiology 
team. In patients with an IVC tumor thrombus extending above the diaphragm, the supradiaphragmatic IVC was dissected by blunt and sharp dissections through a vertical incision in the diaphragm (4). The supradiaphragmatic IVC was then encircled and controlled by a tape. When an IVC tumor thrombus had extended into the RA, a median sternotomy was made, and cannulations of the ascending aorta, superior vena cava, and right femoral vein were carried out to perform extracorporeal circulation (ECC). For patients who were not eligible or unwilling to undergo $\mathrm{LR}$, locoregional therapies including TACE, radiotherapy (RT), and percutaneous ablative therapy/ethanol injection (PAT) were discussed and offered to the patients. Patients with a poor general condition or poor liver function at the time of presentation were only offered the BSC.

\section{Follow-up}

Patients who underwent LR were followed-up once every 3 to 4 months until death or dropout from the followup program. When recurrent HCC was diagnosed, the patients were actively treated with radiofrequency ablation, percutaneous ethanol injection, transarterial chemoembolization, or repeat LR, based on the general condition of the patient, the underlying liver functional status, and the number and location of HCC recurrences. A diagnosis of recurrence of HCC was based on CT and/ or MRI and a raised serum $\alpha$-fetoprotein (AFP) level. Patients who underwent TACE, TACE-RT, PAT or BSC were followed-up once every 6 to 8 weeks. Overall survival (OS) was defined as the interval from the date of receiving the first treatment to the date of death or the last follow-up. OS was used to evaluate the effectiveness of each treatment. This study was censored on June 1, 2018.

\section{Statistical analysis}

For comparisons between baseline variables, the Student's $t$-test was used for continuous variables and the $\chi^{2}$ test for categorical variables. Survival curves and univariate analysis were conducted using the Kaplan-Meier method, and the differences were analyzed using the log-rank test. The significant prognostic factors on univariate analysis $(\mathrm{P}<0.05)$ were subjected to multivariate analysis using the Cox proportional hazards regression model. The time-dependent areas under the receiver operating characteristic curve (AUC) of each point were measured from 10 to 60 months, reflecting their performances in predicting the OS at various time points. The abilities of the different systems in predicting prognosis were compared by the time-dependent AUC and OS curves for each score. Harrell's C-index was used to measure the discriminatory ability of different prognostic systems (22). All the reported $\mathrm{P}$ values were twosided. A significance level of 0.05 was applied throughout. The statistical analyses were performed using the $\mathrm{R}$ statistical package, Version 3.4.3 (R Development Team, Vienna, Austria).

\section{Results}

\section{Patient characteristics}

As shown in Figure S1, during the study period, a total of 5,600 patients with HCC were registered between 2008 to 2016, among which 625 patients (11.2\%) were accompanied with HVTT. Of these, 437 HCC patients with HVTT who met the inclusion criterion were included in the study. The remaining 188 patients were excluded because of poor liver function, extrahepatic metastasis, and missing data.

The baseline characteristics of the patients in the different groups are listed in Table 2. The numbers (percentages) of patients with types I, II, and III HVTT were $146(33.4 \%), 143(32.7 \%)$, and $148(33.9 \%)$, respectively. The type II and III HVTT groups were more likely to have higher rates of $\mathrm{HBV}$ infection, bigger tumors, multiple tumors, coexistence of PVTT, and high levels of carcinoembryonic antigen, total bilirubin, and direct bilirubin when compared to the type I HVTT group. There were also significant differences in the initial treatment modality among the different types of HVTT groups $(\mathrm{P}<0.001)$. The type I HVTT group had a higher rate of surgical treatment, while the type II and III HVTT groups were more likely to undergo TACE treatment. However, there were no significant differences in anti-viral treatment, satellite nodules, lymph node invasion, ascites, and AFP levels among the groups with different types of HVTT. Most patients were male and HBV was the commonest etiological cause of HCC.

\section{Survival analysis}

At a median follow up of 8.4 months (range, 5-68 months), 175 patients $(40.0 \%)$ had died. The 1-, 2-, and 3-year OS rates of all the patients were $56.0 \%, 31.3 \%$, and $12.4 \%$, respectively. The median OS time was 15 months.

The detailed survival outcomes of the groups of patients 
Table 2 Patients characteristics of different types of HVTT (n=437)

\begin{tabular}{|c|c|c|c|c|}
\hline Variables & Type | $(n=146)$ & Type $\|(n=143)$ & Type III (n=148) & $P$ value \\
\hline Sex & & & & 0.945 \\
\hline Male & $129(88.4)$ & $128(89.5)$ & $131(88.5)$ & \\
\hline Female & $17(11.6)$ & $15(10.5)$ & $17(11.5)$ & \\
\hline Anti-viral treatment, yes & $22(15.1)$ & $18(12.6)$ & $19(12.8)$ & 0.793 \\
\hline Tumor diameter, $\mathrm{cm}$ & & & & 0.309 \\
\hline$<5$ & $18(12.3)$ & $15(10.5)$ & $10(6.8)$ & \\
\hline $5-10$ & $75(51.4)$ & $76(53.1)$ & $71(48.0)$ & \\
\hline Multi & $17(11.6)$ & $31(21.7)$ & $38(25.7)$ & \\
\hline Single & $129(88.4)$ & $112(78.3)$ & $110(74.3)$ & \\
\hline Tumor encapsulation & & & & $0.019^{*}$ \\
\hline No & $17(11.6)$ & $11(7.7)$ & $20(13.5)$ & \\
\hline Incomplete & $100(68.5)$ & $80(55.9)$ & $91(61.5)$ & \\
\hline Complete & $29(19.9)$ & $52(36.4)$ & $37(25.0)$ & \\
\hline Satellite nodules, yes & $51(34.9)$ & $42(29.4)$ & $60(40.5)$ & 0.136 \\
\hline Lymph node invasion, yes & $20(13.7)$ & $24(16.8)$ & $20(13.5)$ & 0.677 \\
\hline AFP, ng/mL & & & & 0.132 \\
\hline$\geq 400$ & $65(44.5)$ & $77(53.8)$ & $82(55.4)$ & \\
\hline$<400$ & $81(55.5)$ & $66(46.2)$ & $66(44.6)$ & \\
\hline Total bilirubin, $\mu \mathrm{mol} / \mathrm{L}$ & & & & $0.001^{*}$ \\
\hline$\leq 17.1$ & $89(61.0)$ & $82(57.3)$ & $61(41.2)$ & \\
\hline$>17.1$ & $57(39.0)$ & $61(42.7)$ & $87(58.8)$ & \\
\hline ALB, $g / L$ & & & & $0.047^{*}$ \\
\hline$<35$ & $19(13.0)$ & $21(14.7)$ & $9(6.1)$ & \\
\hline$\geq 35$ & $127(87.0)$ & $122(85.3)$ & 139 (93.9) & \\
\hline $\mathrm{ALT}, \mathrm{U} / \mathrm{L}$ & & & & 0.318 \\
\hline$\leq 44$ & $90(61.6)$ & $76(53.1)$ & $82(55.4)$ & \\
\hline$>44$ & $56(38.4)$ & $67(46.9)$ & $66(44.6)$ & \\
\hline
\end{tabular}

Table 2 (continued) 
Table 2 (continued)

\begin{tabular}{|c|c|c|c|c|}
\hline Variables & Type I $(n=146)$ & Type $\|(n=143)$ & Type III (n=148) & $P$ value \\
\hline$\leq 13$ & $101(69.2)$ & $105(73.4)$ & $105(70.9)$ & \\
\hline$>13$ & $45(30.8)$ & $38(26.6)$ & $43(29.1)$ & \\
\hline Platelet count, $\times 10^{9} / \mathrm{L}$ & & & & 0.461 \\
\hline$\geq 100$ & 105 (71.9) & $111(77.6)$ & $114(77.0)$ & \\
\hline Coexistence of PVTT, yes & $51(34.9)$ & $65(45.5)$ & $72(48.6)$ & $0.046^{*}$ \\
\hline Type I & $19(13.0)$ & $25(17.5)$ & $22(14.9)$ & \\
\hline Type II & $21(14.4)$ & $19(13.3)$ & $22(14.9)$ & \\
\hline Surgery & $62(42.5)$ & $45(31.5)$ & $33(22.3)$ & \\
\hline TACE & $50(34.2)$ & $60(42.0)$ & $58(39.2)$ & \\
\hline TACE + RT & $30(20.5)$ & $32(22.4)$ & $34(23.0)$ & \\
\hline BSC & $1(0.7)$ & $2(1.4)$ & $20(13.5)$ & \\
\hline Others & $3(2.1)$ & $4(2.8)$ & $3(2.0)$ & \\
\hline Median OS, months & $27.1(23.2-30.0)$ & $15.0(12.7-17.3)$ & $8.0(6.8-9.2)$ & $<0.001^{*}$ \\
\hline Overall survival (\%) & & & & $<0.001^{*}$ \\
\hline 1-year & 79.5 & 58.6 & 29.1 & \\
\hline
\end{tabular}

Data are presented as median (range) or $\mathrm{n}(\%) .{ }^{*}$, these values indicate significance. HVTT, hepatic vein tumor thrombus; AFP, $\alpha$-fetoprotein; ALB, albumin; ALT, alanine aminotransferase; PVTT, portal vein tumor thrombus; TACE, transcatheter chemoembolization; RT, radiotherapy; $\mathrm{BSC}$, best supportive care.
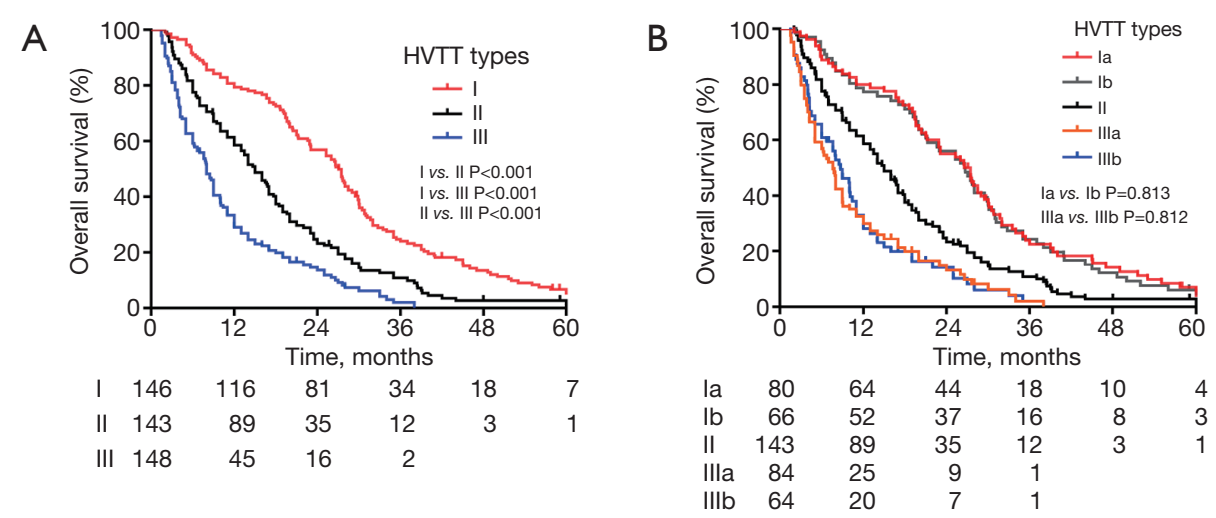

Figure 2 Performance of the HVTT classification. (A) the Kaplan-Meier estimated OS curves according to different HVTT types; (B) the KaplanMeier estimated OS curves according to different HVTT subtypes. HVTT, hepatic vein tumor thrombus; OS, overall survival. 

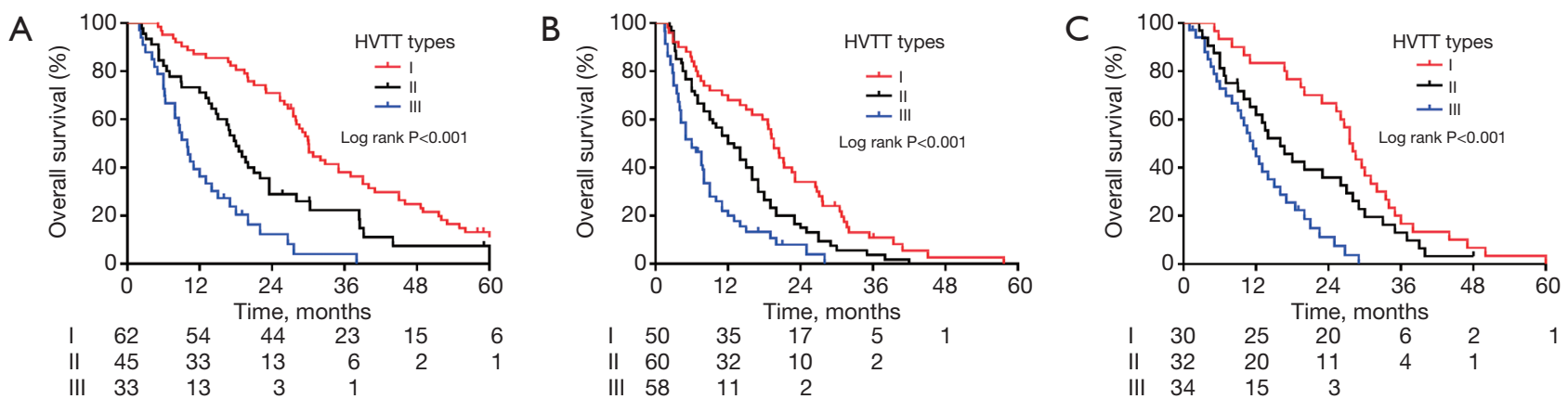

Figure 3 Kaplan-Meier estimated OS curves of HVTT classification among surgical (A), TACE (B), and TACE + RT (C) cohorts. HVTT, hepatic vein tumor thrombus; OS, overall survival; TACE, transcatheter chemoembolization; RT, radiotherapy.

with the different types of HVTT are shown in Table 2. The OS of type III HVTT group was significantly worse than that of type I or II HVTT groups (all $\mathrm{P}<0.001$, Figure $2 A$ ). The OS was also significantly worse in type II HVTT group than type I HVTT group $(\mathrm{P}<0.001$, Figure $2 A)$. There was no significant difference in the OS between type Ia and type Ib HVTT groups $(\mathrm{P}=0.813$, Figure $2 B)$, as well as between type IIIa and type IIIb HVTT groups $(\mathrm{P}=0.812$, Figure $2 B)$. In addition, significant differences were observed between different types of HVTT groups in patients who underwent LR, TACE, or TACE + RT (Figure 3).

Multivariate analysis demonstrated that tumor diameter (HR, 1.511; 95\% CI, 1.035-2.206; $\mathrm{P}=0.032$ ), albumin level (HR, 0.639; 95\% CI, 0.465-1.879; $\mathrm{P}=0.006$ ), coexistence of PVTT (HR, 1.244; 95\% CI, 1.013-1.528; P=0.037), types of HVTT (type II $v s$. type I: HR, 1.672; 95\% CI, 1.303-2.147; type III $v s$. type I: HR, 3.042; 95\% CI, 2.310-4.005; all $\mathrm{P}<0.001$ ), treatment modality (TACE $v s$. surgery: HR, 2.116; 95\% CI, 1.648-2.717; BSC vs. surgery: HR, 4.753; 95\% CI, 2.775-8.140; all $\mathrm{P}<0.001$ ) were risk factors of OS (Table 3).

\section{Predictive accuracy of the HVTT staging system compared with the commonly used international staging systems}

Kaplan-Meier curves were generated for the CLIP, TNM staging, JIS, CUPI, and Vv classification systems (Figure $4 A, B, C, D, E)$. All the five systems showed overlapping survival curves, with no clearly defined prognostic strata. The time-dependent-ROC curve area analysis was used to determine which staging systems were good at predicting the OS. As shown in Figure 4F, for the new HVTT classification, the median time-dependent
AUC was 0.754 (range, 0.716-0.796). The specific AUC values at 1-, 2-, 3-, and 5-year were presented in Table 4. The results showed that the predicting capacity of the new HVTT classification was better than any of the other staging systems, including the CLIP, TNM staging, JIS, CUPI, and $V_{v}$ classification. In addition, the HVTT classification displayed a better C-index (0.724; 0.683-0.765) in predicting OS than the other staging systems (Table S1).

\section{Discussion}

Macrovascular invasion, including PVTT, HVTT, and IVCTT, has been recognized as one of the most significant poor risk factors for HCC patients $(23,24)$. No worldwide consensus exists on the management of HCC accompanied with macrovascular invasion. Various treatments including surgery, TACE, and TACE-RT, have been proposed to treat PVTT. Our team has established a new classification of PVTT to better stratify and predict prognosis in these patients (19,20,25-27). Even less is known about HVTT, probably because of the relative rarity of HVTT compared with PVTT in patients with HCC. Two large sample-size studies from China and Japan concluded that hepatic resection with removal of tumor thrombus was the treatment of choice in selected HVTT patients with resectable diseases, good general conditions and good liver functions $(7,13)$. For patients who are not candidates for LR, non-surgical treatments, including TACE, radiotherapy, and/or systemic chemotherapy have been recommended (8-11). However, the optimal choice for therapeutic options for patients with unresectable diseases is controversial. This study was conducted to establish a new classification of HCC with HVTT to better stratify and 
Table 3 Univariate and multivariable Cox regression analysis of OS in all HVTT patients $(n=437)$

\begin{tabular}{|c|c|c|c|c|c|c|}
\hline Variables & \multicolumn{3}{|c|}{ Univariate analysis } & \multicolumn{3}{|c|}{ Multivariate analysis } \\
\hline Age, $>50$ vs. $\leq 50$, years & 0.002 & $1.001(0.995-1.008)$ & 0.563 & NA & NA & NA \\
\hline Sex, male vs. female & -0.227 & $0.797(0.585-1.084)$ & 0.149 & NA & NA & NA \\
\hline Aetiology, HBV, yes vs. no & 0.096 & $1.101(0.830-1.459)$ & 0.503 & NA & NA & NA \\
\hline \multicolumn{7}{|l|}{ Tumor diameter, cm } \\
\hline $5-10$ vs. $<5$ & 0.403 & $1.496(1.049-2.133)$ & $0.026^{*}$ & 0.295 & $1.342(0.930-1.938)$ & 0.116 \\
\hline$>10$ vs. $<5$ & 0.530 & $1.699(1.179-2.449)$ & $0.004^{*}$ & 0.413 & $1.511(1.035-2.206)$ & $0.032^{*}$ \\
\hline Tumor number, multi vs. single & 0.253 & $1.288(1.006-1.649)$ & $0.044^{*}$ & 0.024 & $1.024(0.794-1.322)$ & 0.854 \\
\hline Complete vs. no & 0.082 & $1.085(0.769-1.530)$ & 0.640 & NA & NA & NA \\
\hline Satellite nodules, yes vs. no & 0.031 & $1.031(0.842-1.264)$ & 0.763 & NA & NA & NA \\
\hline Lymph node invasion, yes vs. no & 0.017 & $1.017(0.773-1.338)$ & 0.905 & NA & NA & NA \\
\hline Ascites, yes vs. no & 0.044 & $1.044(0.785-1.388)$ & 0.764 & NA & NA & NA \\
\hline Child-Pugh grade, B vs. A & 0.058 & $1.059(0.65-1.723)$ & 0.815 & NA & NA & NA \\
\hline AFP, $\geq 400$ vs. $<400, \mathrm{ng} / \mathrm{mL}$ & 0.237 & $1.267(1.043-1.539)$ & $0.017^{*}$ & 0.168 & $1.183(0.966-1.449)$ & 0.104 \\
\hline Total bilirubin, $>17.1$ vs. $\leq 17.1, \mu \mathrm{mol} / \mathrm{L}$ & 0.027 & $1.027(0.845-1.249)$ & 0.786 & NA & NA & NA \\
\hline ALB, $\geq 35$ vs. $<35, \mathrm{~g} / \mathrm{L}$ & -0.435 & $0.648(0.474-0.884)$ & $0.006^{*}$ & -0.447 & $0.639(0.465-1.879)$ & $0.006^{*}$ \\
\hline \multicolumn{7}{|l|}{ HVTT classification } \\
\hline Type II vs. type I & 0.586 & $1.796(1.408-2.290)$ & $<0.001^{*}$ & 0.514 & $1.672(1.303-2.147)$ & $<0.001^{*}$ \\
\hline Type III vs. type I & 1.222 & $3.393(2.630-4.377)$ & $<0.001^{*}$ & 1.112 & $3.042(2.310-4.005)$ & $<0.001^{\star}$ \\
\hline \multicolumn{7}{|l|}{ Treatment modality } \\
\hline TACE vs. surgery & 0.807 & $2.240(1.753-2.863)$ & $<0.001^{*}$ & 0.750 & 2.116 (1.648-2.717) & $<0.001^{*}$ \\
\hline TACE + RT vs. surgery & 0.273 & $1.314(0.998-1.731)$ & 0.052 & 0.044 & 1.045 (0.785-1.393) & 0.761 \\
\hline BSC vs. surgery & 2.248 & 9.464 (5.669-15.79) & $<0.001^{*}$ & 1.559 & 4.753 (2.775-8.140) & $<0.001^{*}$ \\
\hline
\end{tabular}

*, these values indicate significance. HVTT, hepatic vein tumor thrombus; AFP, $\alpha$-fetoprotein; ALB, albumin; ALT, alanine aminotransferase; PVTT, portal vein tumor thrombus; TACE, transcatheter chemoembolization; RT, radiotherapy; BSC, best supportive care; NA, not applicable.

refine HCC patients with macroscopic HVTT to compare the effectiveness of the diversity of treatment modalities for the different subgroups of patients.

To our knowledge, our study is the first to correlate OS with a HVTT classification in a large cohort of patients. Survival analysis demonstrated that the type I, II, and III subgroups in the new HVTT classification were distinct and separate, and the extent of HVTT correlated with 
A

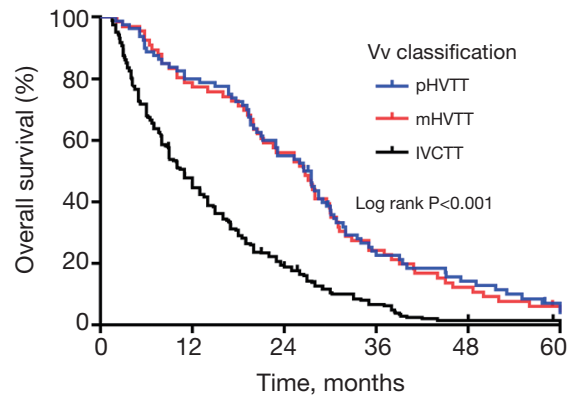

B

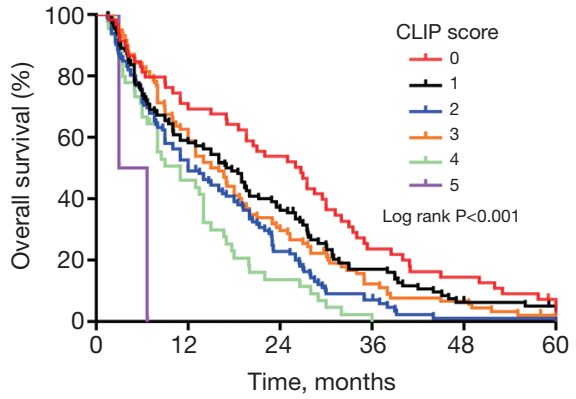

$\mathrm{D}$

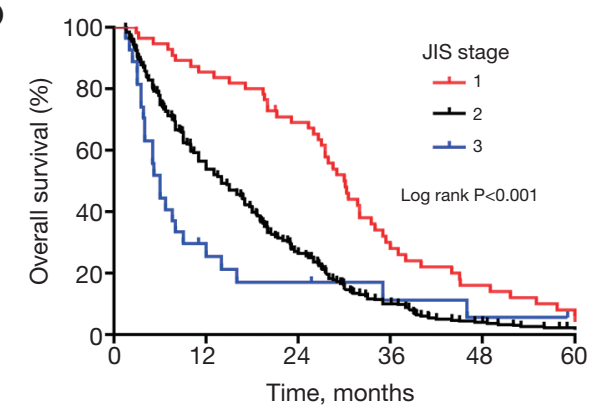

$\begin{array}{lrrrrrr}\text { Stage 1 } & 55 & 47 & 37 & 15 & 8 & 4 \\ \text { Stage 2 } & 355 & 196 & 91 & 31 & 12 & 4 \\ \text { Stage 3 } & 27 & 7 & 4 & 2 & 1 & \end{array}$

$\begin{array}{rrrrrrr}\text { Score 0 } & 59 & 41 & 31 & 13 & 8 & 3 \\ \text { Score 1 } & 110 & 65 & 41 & 16 & 6 & 3 \\ \text { Score 2 } & 112 & 59 & 24 & 7 & 1 & 1 \\ \text { Score 3 } & 109 & 65 & 30 & 11 & 6 & 1 \\ \text { Score 4 } & 45 & 20 & 6 & 1 & & \end{array}$

Score 5

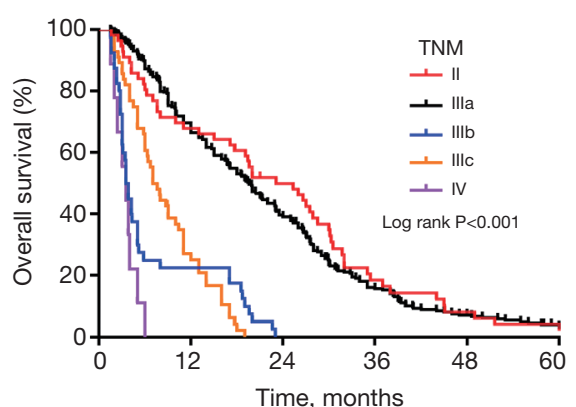

C

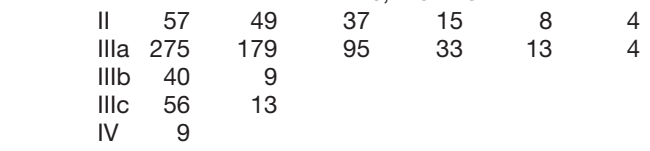

E

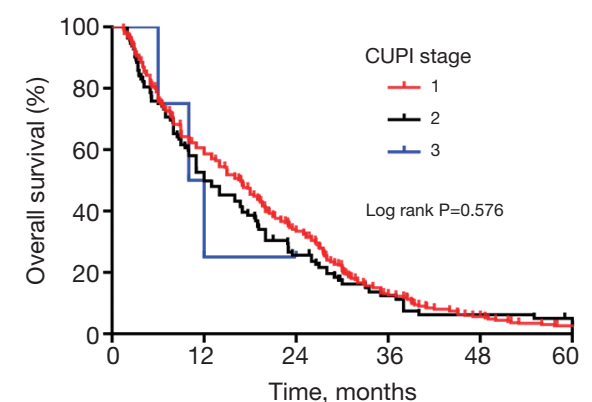

$\begin{array}{lrrrrrr}\text { Stage 1 } & 321 & 191 & 104 & 38 & 16 & 6 \\ \text { Stage 2 } & 112 & 57 & 27 & 10 & 5 & 2 \\ \text { Stage 3 } & 4 & 2 & 1 & & & \end{array}$
$\mathrm{F}$

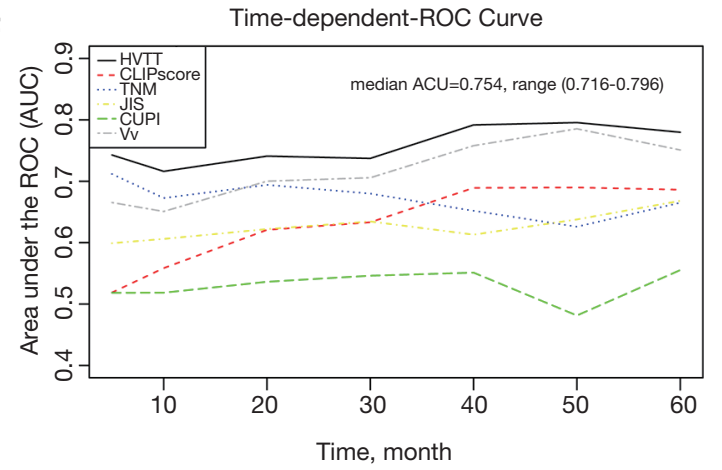

Figure 4 Kaplan-Meier estimated OS curves of the other clinical staging systems in HCC patients with HVTT. (A) The Vv HVTT classification; (B) the Cancer of the Liver Italian Program (CLIP) scoring system; (C) the tumor-node-metastasis TNM stage; (D) the Japan Integrated Staging (JIS) scoring system; (E) the Chinese University Prognostic Index (CUPI) scoring system; (F) time-dependent AUC was measured from 10 to 60 months, reflecting the performance in predicting overall survival at different time points of the different staging systems. HVTT, hepatic vein tumor thrombus; OS, overall survival; HCC, hepatocellular carcinoma; AUC, areas under the receiver operating characteristic curve. 
Table 4 AUC and 95\% CI in the different models for HCC with HVTT

\begin{tabular}{lcccc}
\hline Current model & 1-year & 2-year & 3-year & 5-year \\
\hline HVTT classification & $73.00(68.39-77.61)$ & $73.32(68.50-78.15)$ & $73.63(67.76-79.50)$ & $77.98(68.91-87.05)$ \\
CLIP score & $55.68(50.31-61.05)$ & $63.20(57.52-68.87)$ & $64.84(56.83-72.86)$ & $68.62(51.24-86.00)$ \\
TNM stage & $67.78(63.11-72.45)$ & $69.35(65.55-73.16)$ & $64.41(59.47-69.35)$ & $66.50(53.60-79.41)$ \\
JIS score & $60.63(57.15-64.10)$ & $62.61(58.22-67.00)$ & $59.90(52.69-67.12)$ & $66.81(49.42-84.20)$ \\
CUPI score & $53.76(49.47-58.06)$ & $54.14(49.78-58.49)$ & $52.52(46.28-58.75)$ & $55.53(42.48-68.59)$ \\
Vv classification & $65.85(61.61-70.09)$ & $70.13(65.19-75.08)$ & $69.45(62.22-76.68)$ & $75.09(60.73-89.46)$ \\
\hline
\end{tabular}

HVTT, hepatic vein tumor thrombus; CLIP, Cancer of the Liver Italian Program score; JIS, Japanese Integrated Scoring; CUPI, Chinese University Prognostic Index.

OS. Multivariate analysis also confirmed the new HVTT classification to be a significant risk factor of OS. This new classification divided HVTT into three types which were easy to differentiate by radiologic imagings. The new HVTT classification is practical and can easily use in clinical practice

The Kaplan-Meier curves and time-dependent-ROC curve area analysis were generated to determine the prognostic strata ability of the CLIP, TNM staging, JIS, CUPI, and $V_{v}$ classification systems. The $V_{v}$ classification was proposed to be specific for HCC patients with HVTT who underwent hepatic resection, which showed the unsatisfactory discriminatory ability for prognosis. The study only included 13 HCC patients with IVCTT (7.0\%), which is unable to subdivide these patients into different types. In addition, $\mathrm{Li}$ et al. (28) proposed a new classification for surgical guidance in HCC with IVCTT, which classified IVCTT into three types: type I IVCTT (10 patients) was posterior to the liver and below the diaphragm; type II IVCTT ( 2 patients) was above the diaphragm but still outside the atrium; and type III IVCTT (1 patient) was above the diaphragm and in the RA. However, this IVCTT classification emphasized its guidance to surgery without exploring the prognostic strata ability. The new HVTT classification was more authentic and reasonable to take both anatomical and prognosis analysis into consideration. Furthermore, this study showed the inferiority of the commonly used international systems for HCC patients with HVTT compared to the new HVTT classification. The CLIP score was established based on a cohort of only 12 patients $(2.8 \%)$ who underwent surgical resection. The small sample size may compromise the objectivity and reliability of the scoring system. The CUPI system was based on a cohort composed of a large proportion of patients who underwent only the BSC (29), which is not good in assessing patients who undergo potentially curative treatment. The TNM staging system contains only tumor factors and is restricted to patients who underwent surgical resection. This limits its suitability for application in patients who are not surgical candidates. The JIS system was constructed in Japan based on small sample size, and it needs to be validated by further international studies for use in clinical hepatology (30). All these scoring and staging systems cannot be used to stratify the extent of HVTT in predicting prognosis. The new HVTT classification was established based on a large cohort of patients who underwent different treatments (LR, TACE, TACERT, BSC). This study showed the system can be used in HVTT patients who underwent surgical and non-surgical treatments, and it can be used to compensate for the deficiencies of the other staging systems for this particular group of patients.

The limitations of this study are: first, this study was performed in China with a high proportion of HBV-related HCC. The results should be validated using an international database; second, this is a retrospective study which has its inherent defects and potential biases; third, this is a single center study and the results need to be validated in external cohorts from other centers; fourth, this new classification considers only the type of HVTT, without considering liver function or other tumor-related factors.

In conclusion, a new and practical HVTT classification was established. It can be used to predict prognosis of HCC patients with HVTT who underwent different treatment modalities. This HVTT classification demonstrated a better capacity to stratify the extent of HVTT and to predict 
prognosis when compared with other staging systems. It can be used to supplement the other commonly used international HCC classification and scoring systems.

\section{Acknowledgments}

Funding: This work was supported by the Key Project of Natural Science Foundation of China (No. 81730097); grants from the Science Fund for Creative Research Groups (No. 81521091); the Chang Jiang Scholars Programme [2013] of China Ministry of Education; the National Key Basic Research Programme "973 Project" (No. 2015CB554000); the National Natural Science Foundation of China (No. 81602523), the Shanghai Municipal Health Bureau (No. SHDC12015106); and the Shanghai Science and Technology Committee (No. 134119a0200).

\section{Footnote}

Reporting Checklist: The authors have completed the STROBE reporting checklist. Available at http://dx.doi. org/10.21037/hbsn.2019.10.07

Data Sharing Statement: Available at http://dx.doi. org/10.21037/hbsn.2019.10.07

Conflicts of Interest: All authors have completed the ICMJE uniform disclosure form (available at http://dx.doi. org/10.21037/hbsn.2019.10.07). Dr. WYL serves as an unpaid editorial board member of Hepatobiliary Surgery and Nutrition. The other authors have no conflicts of interest to declare.

Ethical Statement: The authors are accountable for all aspects of the work in ensuring that questions related to the accuracy or integrity of any part of the work are appropriately investigated and resolved. The present study was performed in accordance with the Declaration of Helsinki (as revised in 2013). The study was approved by the Eastern Hepatobiliary Surgery Hospital Research Ethics Committee (No. EHBHKY-2017-002-032). Informed consent was obtained from all the patients prior to treatment, and for their data to be used for research purposes.

Open Access Statement: This is an Open Access article distributed in accordance with the Creative Commons Attribution-NonCommercial-NoDerivs 4.0 International License (CC BY-NC-ND 4.0), which permits the non- commercial replication and distribution of the article with the strict proviso that no changes or edits are made and the original work is properly cited (including links to both the formal publication through the relevant DOI and the license). See: https://creativecommons.org/licenses/by-nc-nd/4.0/.

\section{References}

1. Marrero JA, Kulik LM, Sirlin CB, et al. Diagnosis, Staging, and Management of Hepatocellular Carcinoma: 2018 Practice Guidance by the American Association for the Study of Liver Diseases. Hepatology 2018;68:723-50.

2. Omata M, Cheng AL, Kokudo N, et al. Asia-Pacific clinical practice guidelines on the management of hepatocellular carcinoma: a 2017 update. Hepatol Int 2017;11:317-70.

3. Wang Y, Yuan L, Ge RL, et al. Survival benefit of surgical treatment for hepatocellular carcinoma with inferior vena cava/right atrium tumor thrombus: results of a retrospective cohort study. Ann Surg Oncol 2013;20:914-22.

4. Mizuno S, Kato H, Azumi Y, et al. Total vascular hepatic exclusion for tumor resection: a new approach to the intrathoracic inferior vena cava through the abdominal cavity by cutting the diaphragm vertically without cutting the pericardium. J Hepatobiliary Pancreat Sci 2010;17:197-202.

5. Palmer DH. Sorafenib in advanced hepatocellular carcinoma. N Engl J Med 2008;359:2498; author reply 2498-9.

6. Kokudo T, Hasegawa K, Yamamoto S, et al. Surgical treatment of hepatocellular carcinoma associated with hepatic vein tumor thrombosis. J Hepatol 2014;61:583-8.

7. Kokudo T, Hasegawa K, Matsuyama Y, et al. Liver resection for hepatocellular carcinoma associated with hepatic vein invasion: A Japanese nationwide survey. Hepatology 2017;66:510-7.

8. Kim HC, Lee JH, Chung JW, et al. Transarterial Chemoembolization with Additional Cisplatin Infusion for Hepatocellular Carcinoma Invading the Hepatic Vein. J Vasc Interv Radiol 2013;24:274-83.

9. Chung SM, Yoon CJ, Lee SS, et al. Treatment outcomes of transcatheter arterial chemoembolization for hepatocellular carcinoma that invades hepatic vein or inferior vena cava. Cardiovasc Intervent Radiol 2014;37:1507-15.

10. Zeng $Z$, Fan J, Tang $Z$, et al. A comparison of treatment combinations with and without radiotherapy for hepatocellular carcinoma with portal vein and/or inferior 
vena cava tumor thrombus. Int J Radiat Oncol Biol Phys 2005;61:432-43.

11. Murakami E, Aikata H, Miyaki D, et al. Hepatic arterial infusion chemotherapy using 5-fluorouracil and systemic interferon-alpha for advanced hepatocellular carcinoma in combination with or without three-dimensional conformal radiotherapy to venous tumor thrombosis in hepatic vein or inferior vena cava. Hepatol Res 2012;42:442-53.

12. Zhang XP, Liu YC, Chen ZH, et al. Postoperative Adjuvant Transarterial Chemoembolization Improves Outcomes of Hepatocellular Carcinoma Associated with Hepatic Vein Invasion: A Propensity Score Matching Analysis. Ann Surg Oncol 2019;26:1465-73.

13. Chen ZH, Zhang XP, Wang K, et al. Liver resection versus transcatheter arterial chemoembolization for the treatment of patients with hepatocellular carcinoma and hepatic vein or inferior vena cava tumor thrombus: A propensity score matching analysis. Hepatol Res 2019;49:441-52.

14. Chun YH, Kim SU, Park JY, et al. Prognostic value of the 7 th edition of the AJCC staging system as a clinical staging system in patients with hepatocellular carcinoma. Eur J Cancer 2011;47:2568-75.

15. Kudo M, Chung H, Osaki Y. Prognostic staging system for hepatocellular carcinoma (CLIP score): its value and limitations, and a proposal for a new staging system, the Japan Integrated Staging Score (JIS score). J Gastroenterol 2003;38:207-15

16. Leung TW, Tang AM, Zee B, et al. Construction of the Chinese University Prognostic Index for hepatocellular carcinoma and comparison with the TNM staging system, the Okuda staging system, and the Cancer of the Liver Italian Program staging system: a study based on 926 patients. Cancer 2002;94:1760-9.

17. Llovet JM, Bru C, Bruix J. Prognosis of hepatocellular carcinoma: the BCLC staging classification. Semin Liver Dis 1999;19:329-38.

18. Zhang Z, Lai E, Zhang C, et al. The strategies for treating primary hepatocellular carcinoma with portal vein tumor thrombus. Int J Surg 2015;20:8-16.

19. Shi J, Lai E, Li N, et al. A new classification for hepatocellular carcinoma with portal vein tumor thrombus. J Hepatobiliary Pancreat Sci 2011;18:74-80.

20. Shi J, Lai E, Li N, et al. Surgical treatment of hepatocellular carcinoma with portal vein tumor thrombus. Ann Surg Oncol 2010;17:2073-80.

21. Kasai Y, Hatano E, Seo S, et al. Proposal of selection criteria for operative resection of hepatocellular carcinoma with inferior vena cava tumor thrombus incorporating hepatic arterial infusion chemotherapy. Surgery 2017;162:742-51.

22. Harrell FE Jr, Lee KL, Mark DB. Multivariable prognostic models: issues in developing models, evaluating assumptions and adequacy, and measuring and reducing errors. Stat Med 1996;15:361-87.

23. Pawlik TM, Poon RT, Abdalla EK, et al. Hepatectomy for hepatocellular carcinoma with major portal or hepatic vein invasion: results of a multicenter study. Surgery 2005;137:403-10.

24. Zhang XP, Gao YZ, Chen ZH, et al. An Eastern Hepatobiliary Surgery Hospital/Portal Vein Tumor Thrombus Scoring System as an Aid to Decision Making on Hepatectomy for Hepatocellular Carcinoma Patients With Portal Vein Tumor Thrombus: A Multicenter Study. Hepatology 2019;69:2076-90.

25. Wang K, Guo WX, Chen MS, et al. Multimodality Treatment for Hepatocellular Carcinoma With Portal Vein Tumor Thrombus: A Large-Scale, Multicenter, Propensity Mathching Score Analysis. Medicine (Baltimore) 2016;95:e3015.

26. Li XL, Guo WX, Hong XD, et al. Efficacy of the treatment of transarterial chemoembolization combined with radiotherapy for hepatocellular carcinoma with portal vein tumor thrombus: A propensity score analysis. Hepatol Res 2016;46:1088-98.

27. Kokudo T, Hasegawa K, Matsuyama Y, et al. Survival benefit of liver resection for hepatocellular carcinoma associated with portal vein invasion. J Hepatol 2016;65:938-43.

28. Li AJ, Zhou WP, Lin C, et al. Surgical treatment of hepatocellular carcinoma with inferior vena cava tumor thrombus: a new classification for surgical guidance. Hepatobiliary Pancreat Dis Int 2013;12:263-9.

29. Kinoshita A, Onoda H, Fushiya N, et al. Staging systems for hepatocellular carcinoma: Current status and future perspectives. World J Hepatol 2015;7:406-24.

30. Kudo M, Chung H, Haji S, et al. Validation of a new prognostic staging system for hepatocellular carcinoma: the JIS score compared with the CLIP score. Hepatology 2004;40:1396-405.

Cite this article as: Chen $\mathrm{ZH}$, Wang $\mathrm{K}$, Zhang XP, Feng JK, Chai ZT, Guo WX, Shi J, Wu MC, Lau WY, Cheng SQ. A new classification for hepatocellular carcinoma with hepatic vein tumor thrombus. HepatoBiliary Surg Nutr 2020;9(6):717-728. doi: 10.21037/hbsn.2019.10.07 